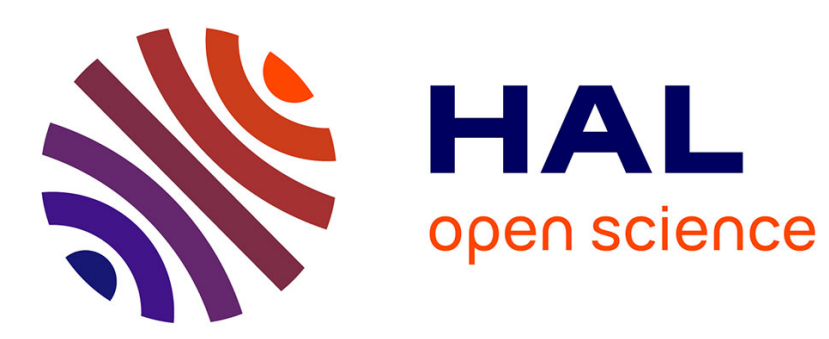

\title{
Wetting and multistable anchorings in nematics
}

B. Jérome, P. Pieranski

\section{- To cite this version:}

B. Jérome, P. Pieranski. Wetting and multistable anchorings in nematics. Journal de Physique, 1988, 49 (9), pp.1601-1613. 10.1051/jphys:019880049090160100 . jpa-00210841

\section{HAL Id: jpa-00210841 https://hal.science/jpa-00210841}

Submitted on 1 Jan 1988

HAL is a multi-disciplinary open access archive for the deposit and dissemination of scientific research documents, whether they are published or not. The documents may come from teaching and research institutions in France or abroad, or from public or private research centers.
L'archive ouverte pluridisciplinaire HAL, est destinée au dépôt et à la diffusion de documents scientifiques de niveau recherche, publiés ou non, émanant des établissements d'enseignement et de recherche français ou étrangers, des laboratoires publics ou privés. 
Classification

Physics Abstracts

$61.30-69.45$

\title{
Wetting and multistable anchorings in nematics
}

\author{
B. Jérome and P. Pieranski \\ Laboratoire de Physique des Solides, Bât. 510, Faculté des Sciences, 91405 Orsay Cedex, France
}

\begin{abstract}
Résumé. - Dans le cas d'un ancrage multistable, une direction d'ancrage doit être choisie parmi les différentes directions possibles. En utilisant comme exemple l'étalement de gouttes de nématique sur des films de $\mathrm{SiO}$ évaporé sous incidence oblique, nous montrons que cette sélection se fait pendant le mouillage du substrat par le nématique. Nous analysons le processus de mouillage et nous proposons un modèle expliquant plusieurs caractéristiques des textures observées expérimentalement dans des gouttes étalées.
\end{abstract}

\begin{abstract}
In the case of multistable anchorings, the selection between competing anchoring directions must be achieved. Using as an example the spreading of nematics droplets on $\mathrm{SiO}$ films evaporated under oblique incidence, we show that this selection is accomplished during the wetting of the substrate by the nematic. We analyse the process of wetting and propose a model explaining several features of experimentally observed textures in spreaded droplets.
\end{abstract}

\section{Introduction : elements of wetting process.}

Anchoring of nematics on solid substrates, in most cases, is determined during the wetting of the substrate by the nematic liquid.

The wetting process involves several elements (Fig. 1) [1] : (1st) the motion of the contact line $\mathbf{C}$, which, like in a zipper, unites (2nd) the wetting interface (air/nematic) with (3rd) the surface to wet (the solid substrate) and leaves (4th) the wetted surface (with a fixed anchoring) behind.

In spite of the fact that all the first three «input » elements can be controlled before and during the wetting only the third one has attracted so far most

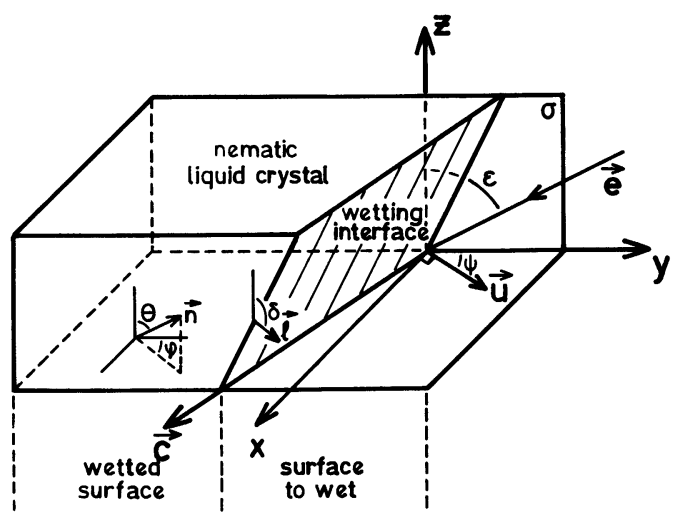

Fig. 1. - Geometry of wetting. $\sigma=$ incidence plane ; $\mathbf{e}=$ direction of evaporation. of the interest; several treatments of the substrate (for example evaporation of an $\mathrm{SiO}$ film on a smooth glass plate), altering its chemical composition, its texture and finally its symmetry on microscopic scale, have been proposed [2].

This element of wetting process deserves fairly such special attention because it is a crucial, «sine qua non » condition of anchoring; the symmetry $\mathrm{G}_{\mathrm{s}}$ of the substrate to wet determines virtually possible types of anchorings, that is to say, stars $\left\{\mathbf{n}_{\alpha}\right\}$ of anchoring directions $\mathbf{n}_{\alpha}=g \mathbf{n}_{1}$, generated by elements $g$ of the group $\mathrm{G}_{\mathrm{s}}$ from an arbitrary vector $\mathbf{n}_{1}$.

However, in the case of multistable anchorings, where the set $\left\{\mathbf{n}_{\boldsymbol{\alpha}}\right\}$ contains more than one vector, selection between the competing anchoring directions must be accomplished.

Our generic idea is that the anchoring selection is achieved during the motion of the contact line - the first element of the wetting process.

The motion of the contact line is characterized by two parameters : the direction $\psi$ and the velocity $u$ (Fig. 1) or the polar $(\delta(u))$ and azimuthal $(\psi)$ angles of the dynamical direction 1 of the wetting interface.

In the present paper we will focus on a special case of $\mathrm{SiO}$ films, where a bistable anchoring occurs $[3,4$, $5]$, and explore the whole sphere of available wetting parameters $(\delta, \psi)$ by studying the spreading of droplets of a nematic E8 [6] on such films. 
We will show that the textures of spreaded droplets are finger-prints of different types of anchorings and prove the reality of anchoring selection during the wetting. We will also develop a model of a mechanism of the anchoring selection and use it to explain these wetting textures.

\section{Experimental.}

\subsection{SURfACE TO WET : SiO FILMS.}

2.1.1 Structure and aligning properties of $\mathrm{SiO}$ films evaporated under oblique incidence. - Films of $\mathrm{SiO}$ evaporated under oblique incidence are highly porous and anisotropic; electron micrographes show that they are made of column-like dendrites which are oblique with respect to the substrate but parallel to the plane of incidence $\sigma$ of the vapour beam [7]. The inclination of dendrites increases with the incidence angle.

More recently, such dendritic structures have been found by numerical simulations in so-called ballistic deposition model by Jullien and Meakin [8]. Let $\rho(\mathbf{r})$ be the local density of the film. One can define a density correlation function

$$
g(\tau)=\langle\rho(\mathbf{r}+\boldsymbol{\tau}) \cdot \rho(\mathbf{r})\rangle
$$

which caracterizes the average anisotropy and inhomogeneity of the film. For above-mentioned dendritic structure, $g(\tau)$ has the symmetry $\mathrm{G}_{\mathrm{s}}=$ $\mathrm{C}_{\mathrm{s}}(E, \sigma)$ where $E$ is the identity and $\sigma$ is the reflection in the plane of incidence.

Following considerations of the introduction, three distinct discret stars $\left\{\mathbf{n}_{\alpha}\right\}$ can be generated from an arbitrary vector $\mathbf{n}_{1}$ by elements of the group $\mathrm{C}_{\mathrm{s}}$ so that three types of anchoring exist for this symmetry :

(a) symmetric tilted:

if $\mathbf{n}_{1}$ is parallel to the mirror plane, $\sigma$ then $\sigma \mathbf{n}_{1}=\mathbf{n}_{1}$ so that $\left\{\mathbf{n}_{\alpha}\right\}=\left\{\mathbf{n}_{1} \equiv-\mathbf{n}_{1}\right\}$;

(b) antisymmetric planar :

if $\mathbf{n}_{1}$ is perpendicular to the mirror plane $\sigma$, then $\boldsymbol{\sigma} \mathbf{n}_{1}=-\mathbf{n}_{1} \equiv \mathbf{n}_{1}$ so that $\left\{\mathbf{n}_{\alpha}\right\}=\left\{\mathbf{n}_{1} \equiv-\mathbf{n}_{1}\right\}$;

(c) asymmetric tilted :

If $\mathbf{n}_{1}$ is oblique with respect to the mirror plane then $\sigma \mathbf{n}_{1}=\mathbf{n}_{2} \neq-\mathbf{n}_{1}$, so that $\left\{\mathbf{n}_{\alpha}\right\}=\left\{\mathbf{n}_{1}, \mathbf{n}_{2}\right\}$. As one of the anchoring direction is chosen by the wetting conditions, the symmetry $\mathrm{G}_{\mathrm{s}}$ of the substrate is broken; so we call this anchoring asymmetric tilted anchoring.

Experimentally, the aligning properties of evaporated $\mathrm{SiO}$ films have been first pointed out by Janning [9]. Guyon et al. [10] have shown that the orientation of a nematic liquid on such films depends on the angle of incidence $\varepsilon$ (Fig. 1) of the vapour beam :
— for grazing incidences $\left(\varepsilon>75^{\circ}\right)$ the anchoring is symmetric tilted;

- for intermediate incidences $\left(40^{\circ}<\varepsilon<75^{\circ}\right)$ the anchoring is antisymmetric planar;

- in the vicinity of normal incidence the anchoring is parallel to the substrate but there is no preferred azimuthal directions (degenerate planar anchoring).

In fact, the transition between the symmetric tilted and antisymmetric planar anchorings is not discontinuous but mediated by the asymmetric tilted anchoring. In the present paper we discuss the wetting of $200 \AA$ thick SiO films where, as reported in reference [5] and shown in more details in the next sections, the asymmetric tilted anchoring occurs as a function of the incidence angle $\varepsilon$ of the vapour beam.

2.1.2 Coating conditions. - In the present study we have first coated microscope slides $(7.5 \mathrm{~cm}$ long) with $\mathrm{SiO}$ films, approximately $200 \AA$ thick, evaporated at a rate of $8 \AA \mathrm{s}^{-1}$ (measured by a quartz microbalance at normal incidence) in vacuum of $10^{-5}$ Torr. The geometry of evaporation is shown in figure 2. The angle of incidence is changed by rotating the slide around the axis $\Delta$; for a given incidence $\varepsilon_{0}$, at point $O$, one end of the slide, the incidence angle $\varepsilon(x)$ is expressed by a distance $x$ from $\mathrm{O}$ on the slide as follows

$$
\varepsilon(x)=\varepsilon_{0}+\arctan \frac{x \cos \varepsilon_{0}}{h+x \sin \varepsilon_{0}} .
$$

Due to the finite size of the $\mathrm{SiO}$ source, the vapour beam incident at point $x$ has a small angular dispersion : $\delta \varepsilon=\frac{d}{h} \approx 2^{\circ}$.

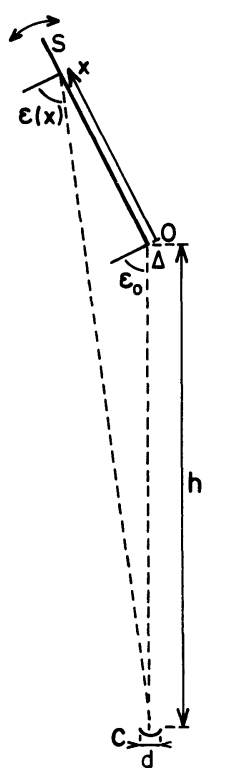

Fig. 2. - Geometry of evaporation : $h=24.5 \mathrm{~cm} ; d$ $1 \mathrm{~cm} ; \mathrm{C}: \mathrm{SiO}$ source ; $\mathrm{S}$ : glass slide. 
Such large slides have been used for experiments on wetting by radial spreading of nematic droplets (Sect. 2.2.1).

The same evaporation conditions have been used to coat $E$-shaped glass plates for measurements of the tilt angle (Sect. 2.2.2).

\subsection{MOTION OF THE CONTACT LINE.}

2.2.1 Spontaneous spreading of droplets. - In order to explore the wetting of $\mathrm{SiO}$ films for many, closely spaced values of the incidence angle $\varepsilon$ and for all possible directions $\psi$ and $\delta$ of the wetting interface, we have used the following method:

droplets of the nematic $\mathrm{E}$, hanging from a teflon capillary tube, have been deposited on the glass slides (coated with $\mathrm{SiO}$ ) at closely spaced distances $x_{i}$. Knowing $x_{i}$, the evaporation incidence angle $\varepsilon\left(x_{i}\right)$ was calculated precisely using equation (2). As the radius of a spreaded droplet $\Delta x$ was typically $2 \mathrm{~mm}$, the corresponding variation of $\varepsilon$ across the droplets varied from $\Delta \varepsilon=0.9^{\circ}$ for $\varepsilon=0^{\circ}$ to $\Delta \varepsilon=$ $0.2^{\circ}$ for $\varepsilon=80^{\circ}$. By this means closely spaced evaporation angles have been sampled.

Such droplets stay approximately circular during their spreading. This means that the direction of motion of the contact line is radial so that all possible directions of motion of the contact line are explored. As the contact angle varies continuously from $\pi$ to almost zero (for a droplet which radius grows from 0 to $2 \mathrm{~mm}$ in few minutes) almost all possible directions $(\delta, \psi)$ of the wetting interface are explored.

In the approximation, where during spreading the droplets keep the shape of a spherical cap, each point $(r, \psi)$ inside the spreaded droplets corresponds to one point $(\delta, \psi)$ on the sphere of wetting conditions ; the relationship between $\delta$ and $r$ can be calculated from the expression of the volume $V$ of a spherical cap :

$$
V=\frac{1}{3} \pi\left(\frac{r(1-\cos \delta)}{\sin \delta}\right)^{3}\left(\frac{3}{1-\cos \delta}-1\right)
$$

where $r$ is the radius of the circular base of the cap and $\delta$ the dynamical contact angle.

2.2.2 Forced wetting. - As we have mentioned in section 1 , instead of the direction $\mathbf{l}$ of the wetting interface, the velocity $\mathbf{u}(u, \psi)$ of the contact line can be considered as the pertinent parameter of the wetting process.

In such a case we have studied forced wetting of SiO films : a teflon capillary tube containing the nematic E8 has been approached to the horizontal glass slide at a distance of about $0.1 \mathrm{~mm}$. Using a microsyringe the nematic was pushed from the tube toward the slide and a circular liquid bridge, of diameter $\approx 2 \mathrm{~mm}$, connecting the tube and the slide was formed by this means. Using the microscope stage, the glass slide was then moved at a well known velocity $\mathrm{u}$ in a given direction $\psi$. Knowing the shape of the moving contact line its local normal direction and velocity can be determined from geometrical consideration.

2.2.3 Wetting of cells limited by two glass plates. As we will see in section 2.3.2 the measurement of the tilt angle requires monodomain samples. This case is opposite to the previous one ; the direction and the velocity of the contact line is fixed during the wetting of both limiting glass plates in order to prevent switching between the anchorings $\mathbf{n}_{1}$ and $\mathbf{n}_{2}$.

\subsection{RESULTS.}

2.3.1 Textures of spreaded droplets; selection of anchoring by wetting. - The spreaded droplets of E8 observed in a polarizing microscope have shown very characteristic textures in their central part $(r \leq 2 \mathrm{~mm})$.

$0^{\circ} \leqslant \varepsilon \leqslant 3^{\circ}$ : the droplet shows a dark cross on a bright background (Fig. 3). The center of the cross coincides with the center of the droplet and its branches are parallel to the polarizers. When the droplet is rotated, the cross keeps its orientation. This texture is characteristic of a splay configuration. The anchoring is planar $\left(\theta=90^{\circ}\right)$ and radial $(\varphi=\psi)$; it depends on the direction $\psi$ but not on the dynamical contact angle $\delta(r)$.

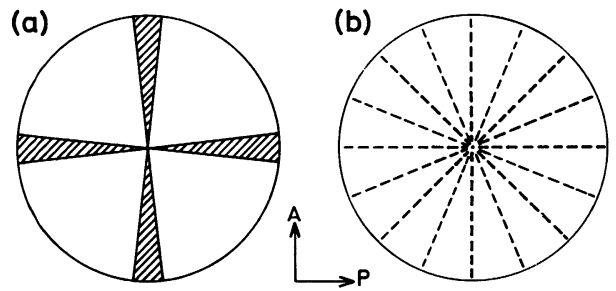

Fig. 3. - Degenerate planar anchoring: a) droplet observed between crossed analyser and polarizer ; b) nematic orientation.

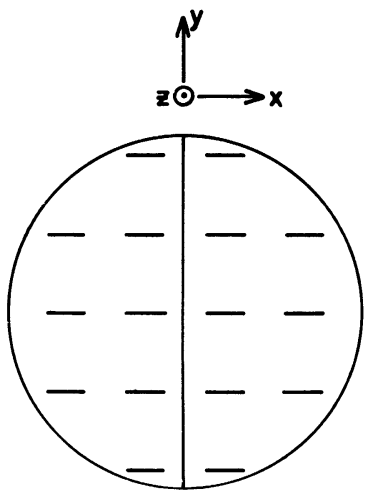

Fig. 4. - Antisymmetric planar anchoring. 

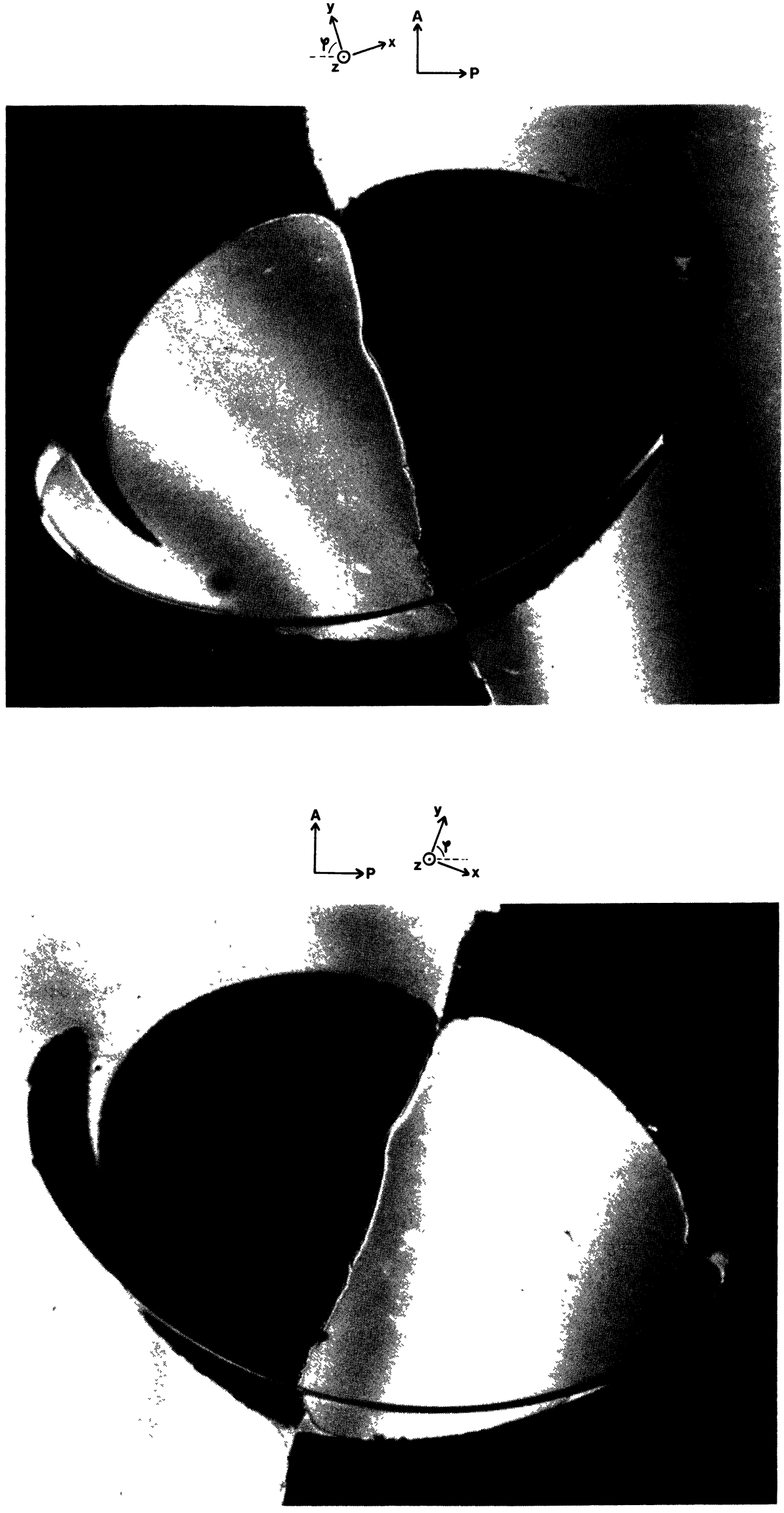

a)

Fig. 5. - Asymmetric tilted anchoring : central part of the droplet ; a) type I ; b) type II. 

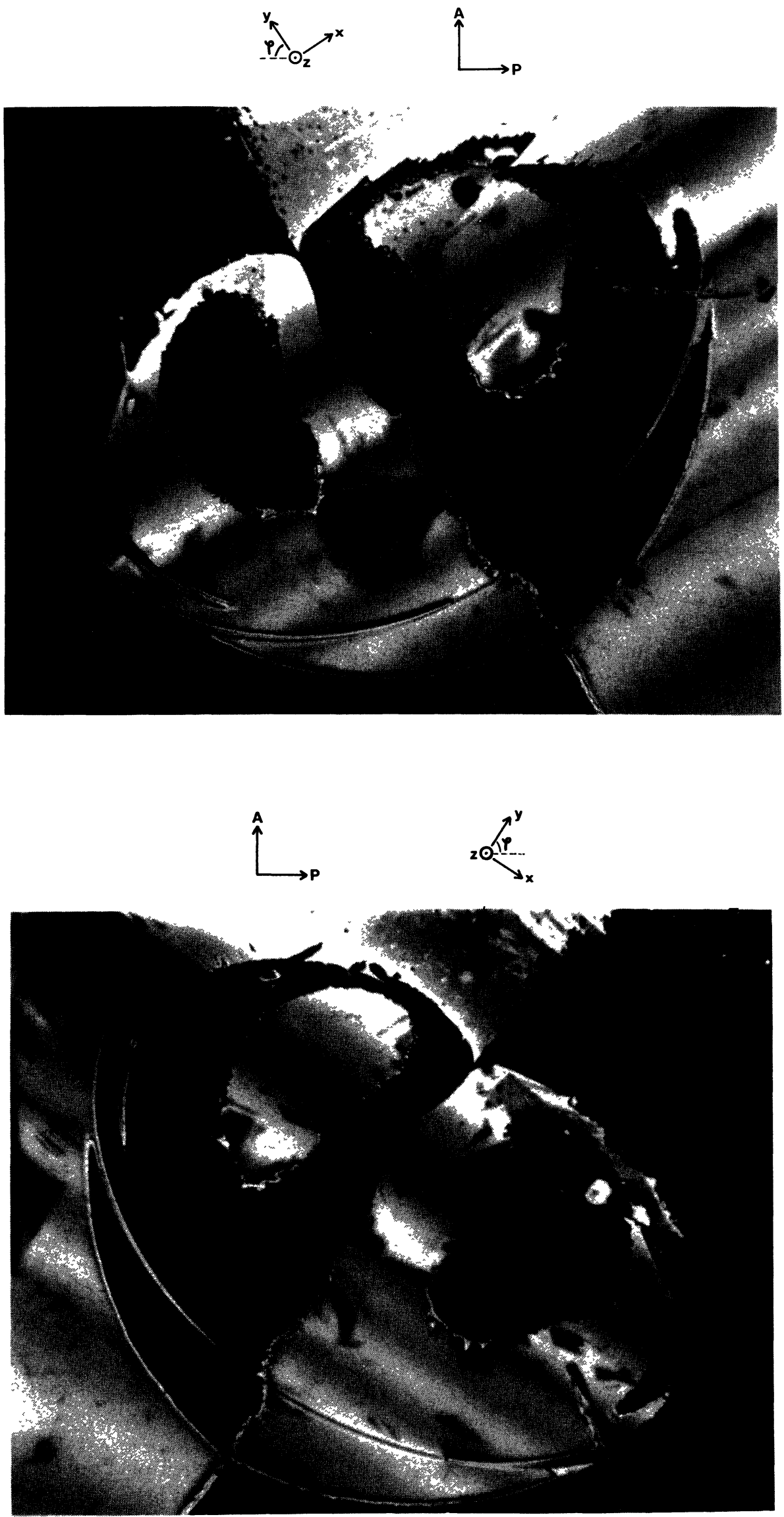

b)

Fig. 5 (continued) 
$3^{\circ} \leqslant \varepsilon \leqslant 11^{\circ}$ : the texture of the droplet is quite confused. One can observe a diametral $\pi$-wall (see below) but extinction of the whole droplet cannot be obtained.

$11^{\circ} \leqslant \varepsilon \leqslant 60^{\circ}$ : when the slide is oriented with the incidence plane of evaporation $\sigma$ parallel to one of the polarizers, the droplet is dark with a diametral bright line parallel to $\sigma$. The anchoring is antisymmetric planar (Fig. 4) ; the bright line is a $\pi$-wall between $\mathbf{n}_{1}$ and $\mathbf{n}_{2}=-\mathbf{n}_{1}$ domains. The selection between $\mathbf{n}_{1}$ and $-\mathbf{n}_{1}$ depends only on the direction $\psi$ but not on the dynamical contact angle $\delta(r)$.

$60^{\circ}<\varepsilon<72^{\circ}$ : the droplets show two kinds of textures (see Fig. 5). The type II occurs only for $66^{\circ}<\varepsilon<68^{\circ}$ and is not as well reproductible as the type I. In this last case the droplet is divided in four uniformly oriented domains by three walls; one is diametral and parallel to $\sigma$ as in the previous case, the other two have the shapes of a cardioid and a crescent.

The anchoring is asymmetric tilted : two different anchorings $\mathbf{n}_{1}$ and $\mathbf{n}_{2}$, images one of the other in the mirror plane, are realized. Their selection depends on both the direction $\psi$ and the contact angle $\delta(r)$ (Fig. 6).

The direction of the anchoring, for example of $\mathbf{n}_{1}$ characterized by the angles $(\theta, \varphi)$ (Fig. 1) depends on the evaporation angle $\varepsilon$. The azimutal

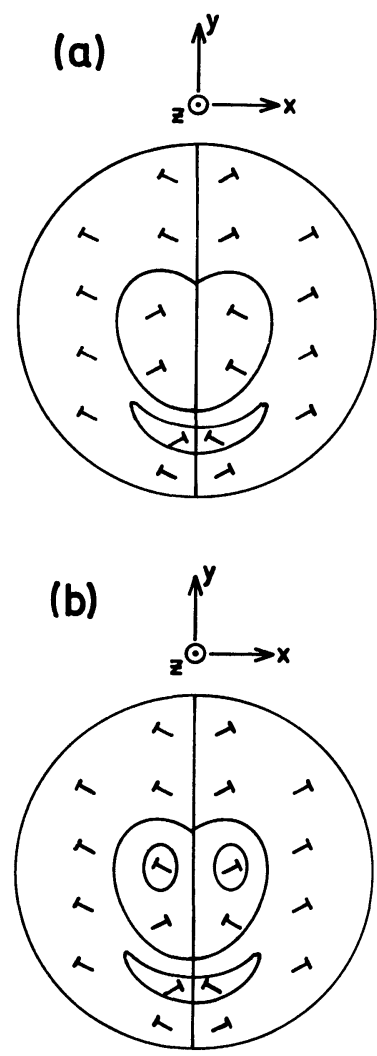

Fig. 6. - Asymmetric tilted anchoring: nematic orientation in the droplet ; a) type I ; b) type II. angle $\varphi$ can be determined precisely by measuring the angle $2 \varphi$ between the two orientations of the droplet corresponding to the extinction of adjacent domains (Fig. 5). As shown in figure 7, $\varphi$ varies continuously between $90^{\circ}$ and $0^{\circ}$ when $\varepsilon$ increases from $60^{\circ}$ to $72^{\circ}$. The variation of the tilt angle is measured in section 2.3.3.

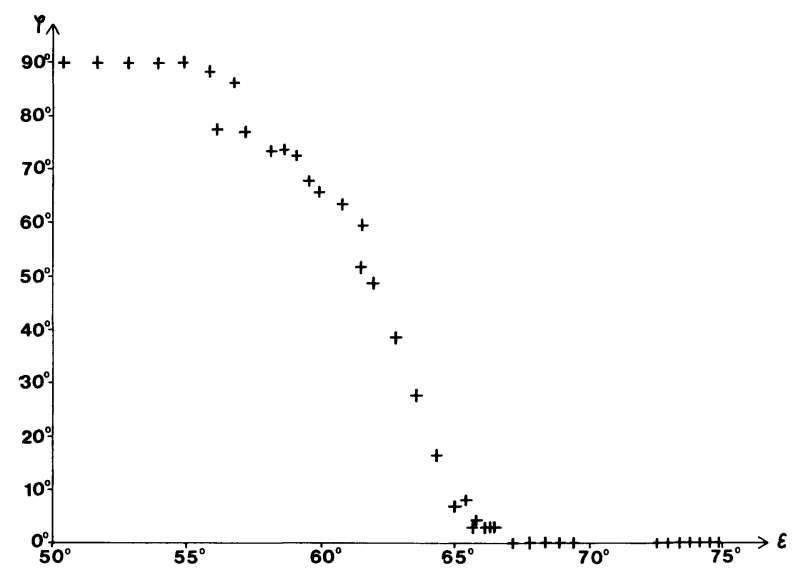

Fig. 7. - Azimuthal orientation $\varphi$ versus incidence angle $\varepsilon$.

$72^{\circ}<\varepsilon<80^{\circ}$ : when the slide is oriented with the incidence plane $\sigma$ parallel to one of the polarizers the droplet appears dark with cardioid and crescentshaped bright lines. The anchoring in this case is symmetric tilted ; the bright lines are $\pi$-walls separating domains with equivalent $\mathbf{n}_{1}$ and $\mathbf{n}_{2}=-\mathbf{n}_{1}$ anchorings (Fig. 8). Their selection depends on both the direction $\psi$ and the contact angle $\delta(r)$.

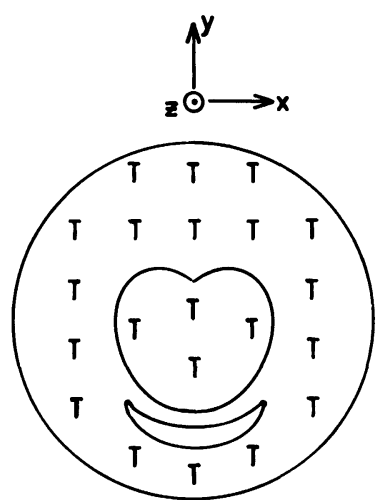

Fig. 8. - Symmetric tilted anchoring : droplet aspect.

Textures in the periphery of droplets $(r \geqslant 2 \mathrm{~mm})$ : in droplets which have been allowed to spread for a long time, the quality of anchoring on their periphery is very poor. The selection of anchoring seems to be not efficient for very small contact 
angles $\delta(r)$ characteristic of such very thin and large droplets ; in those peripheric regions the selection of anchoring seems to be random (presumably related to local inhomogeneities of the $\mathrm{SiO}$ film).

2.3.2 Estimation of the critical velocity. - Using the method described in section 2.2.2, we have determined the velocity $u_{\mathrm{c}}$ corresponding to the switching between anchoring directions $\mathbf{n}_{1}$ and $\mathbf{n}_{2}$ occurring during the creation of the cardioid and crescentshaped walls. It depends on the direction $\psi$ and is the lowest for $\psi=\pi$. The typical value $u_{\mathrm{c}}(\psi=\pi)$ is $200 \mathrm{\mu m} \mathrm{s}^{-1}$.

2.3.3 Measurements of the tilt angle. - The tilt angle $\theta$ of the asymmetric and symmetric tilted anchorings has been measured using samples prepared in the following manner :

- two $E$-shaped glass slides have been evaporated simultaneously; as for a given evaporation angle, the azimutal angle $\varphi$ of anchoring is already known, the slides have been oriented during the evaporation in such a way that one of their anchoring directions is parallel to the three bars of the $E$-shape (Fig. 9a) ;

- evaporated slides have been superposed as shown in figure $9 \mathrm{~b}$ : the slides are separated by $150 \mu \mathrm{m}$ thick spacers and their central bars overlap slightly ;

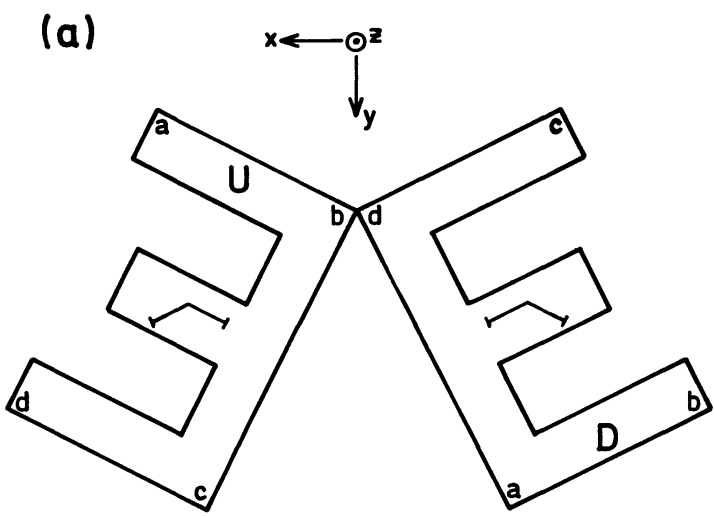

(b)

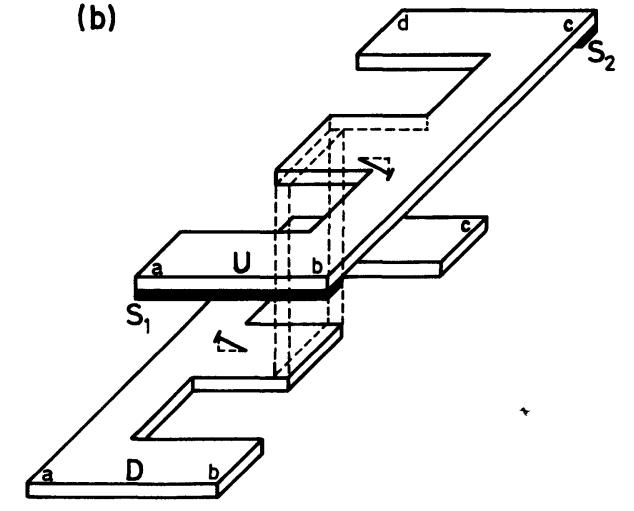

Fig. 9. - Nematic cell : a) evaporation geometry ; b) assembling of the cell $; S_{1}$ and $S_{2}$ : spacers.
- a volume of the nematic, equal to the final volume of the cell, has been injected in the region of overlapping ;

- the upper slide has then been glided slowly on the lower one in order to make the central bars of the two $E$-shapes completely overlap. During this operation the wetting of limiting glass surfaces takes place in a controlled way: the motion of contact lines on both surfaces is such that selected anchorings should be identical.

We have made such cells for eight incidence angles $\varepsilon=60^{\circ}, 61^{\circ}, 64^{\circ}, 67^{\circ}, 70^{\circ}, 72^{\circ}, 78^{\circ}$ and $83^{\circ}$. For $\varepsilon=60^{\circ}$ (antisymmetric planar anchoring), $72^{\circ}$, $78^{\circ}, 83^{\circ}$ (symmetric tilted anchoring) the cells were perfectly monodomain. For $\varepsilon=64^{\circ}, 67^{\circ}$ and $70^{\circ}$ (asymmetric tilted anchoring) we obtained large domains of expected orientation but containing inclusions of twisted texture; the wetting velocity seemed to be not uniform enough to prevent accidental selection of the second available anchoring. For $\varepsilon=61^{\circ}$ the two possible anchorings are very close to each other (the angle between them is $4^{\circ}$ ), and none of them could be selected by our wetting process ; the anchoring was poor and the tilt angle $\theta$ could not be measured.

The cells were introduced in a magnetic field and observed by conoscopy. The cells were rotated in the field in a way to align their optical axis $\mathbf{n}$ with the field $\mathbf{H}$ : when $\mathbf{n}$ is parallel to the field, switching on and off the field does not affect the interference figure. Thus, the values of $\theta$ were measured and are plotted in figure 10.

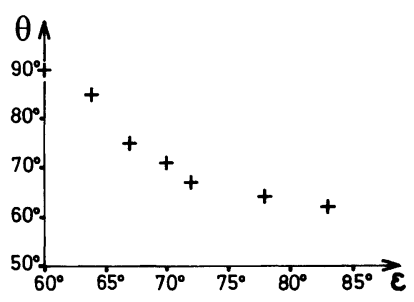

Fig. 10. - Tilt angle $\theta$ versus incidence angle $\varepsilon$.

\section{Mechanism of anchoring selection.}

3.1 SPHERICAL MAPS OF ANCHORING SELECTION. Using equation (3), the textures of spreaded droplets in the plane $(r, \psi)$ can be mapped onto the sphere $(\delta, \psi)$ of the dynamical direction 1 of the wetting interface. The diametral wall $(\psi=0, \pi)$, characteristic of the asymmetric tilted and antisymmetric planar anchorings maps into a great circle $\mathrm{C}_{\sigma}$ parallel to the mirror plane $\sigma$. The cardioid-shaped wall, characteristic of the symmetric and asymmetric tilted anchorings is mapped approximately into an oblique great circle $\mathrm{C}_{\perp}$ perpendicular to $\sigma$. Finally, 
each of the anchoring directions $\mathbf{n}_{\alpha}$ is mapped inside one of the domains on the sphere $(\delta, \psi)$ delimited by the circles $\mathrm{C}_{\sigma}$ and $\mathrm{C}_{\perp}$ (Fig. 11).
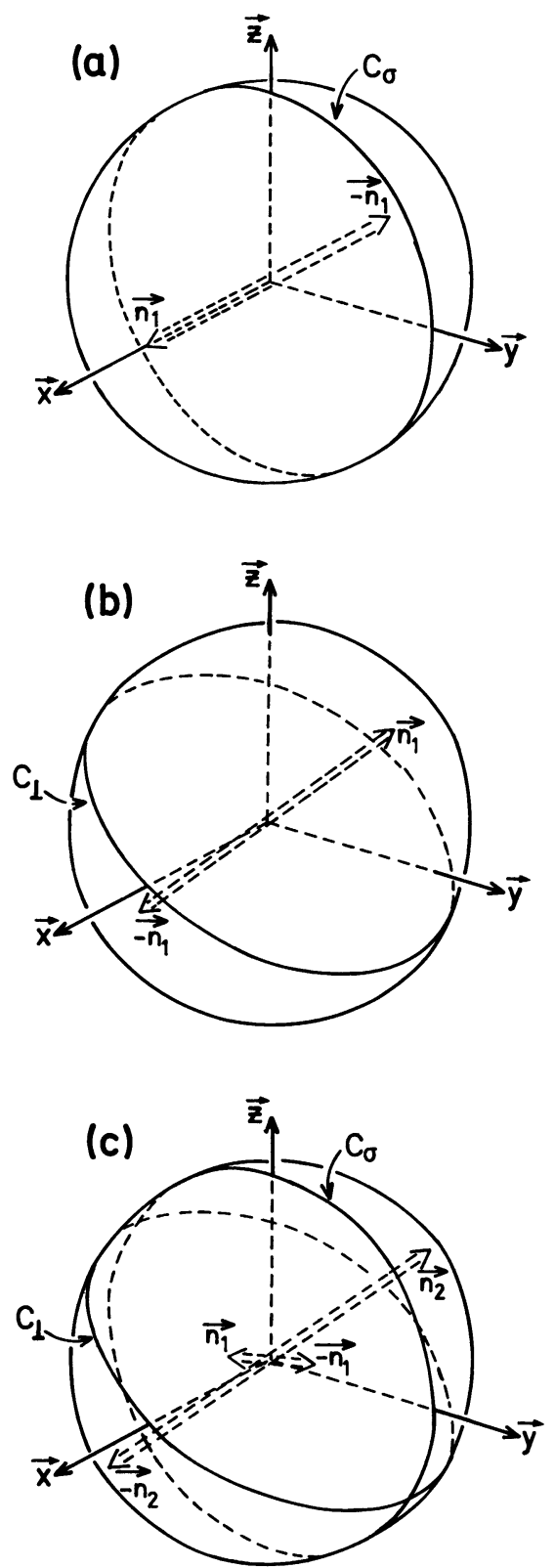

Fig. 11. - Spherical map of anchoring selection : a) antisymmetric planar; b) symmetric tilted; c) asymmetric tilted.

3.2 ANISOTROPIC INTERACTIONS. - Such spherical maps suggest the following mechanism of anchoring selection.

In the case of the nematic E8 the direction $\mathbf{n}_{l}$ of molecules at a free air/nematic interface is normal to it : $\mathbf{n}_{l}=\mathbf{l}$. In the reference frame of the contact line, the flow pattern in the vicinity of the contact line [11] is such that these molecules move toward the contact line and are submitted progres- sively to interactions with the dry and the already wet substrate. Obviously, only the anisotropic parts of these interactions are involved in the anchoring selection. Let us call these anisotropic interactions $V_{\mathrm{D}}\left(\mathbf{n}_{l}, z\right)$ and $V_{\mathrm{w}}\left(\mathbf{n}_{l}, \mathbf{n}_{\alpha}, z\right)$.

Interactions $V_{\mathrm{D}}$ with the dry substrate depend on the orientation $\mathbf{n}_{l}$ of the molecules at the wetting interface and on the distance $z$ from the substrate. Obviously, this part of the interaction must respect the symmetry $\mathrm{G}_{\mathrm{s}}$ of the substrate. The detailed form of $V_{\mathrm{D}}$ is discussed below.

The interaction $V_{\mathrm{w}}$ for the wet substrate depends on the angle between $\mathbf{n}_{l}$ and the direction $\mathbf{n}_{\alpha}$ of the already selected anchoring. In the mechanism of anchoring selection it plays a role of a feedback term if one considers the orientations $\mathbf{n}_{l}$ and $\mathbf{n}_{\alpha}$ respectively as input and output parameters. We will discuss its role in section 3.6.

Our guess is that $V_{\mathrm{D}}(\mathbf{n})$ has valleys with minima located at $\mathbf{n}_{\alpha}$ and that the great circles $\mathrm{C}_{\sigma}$ and $\mathrm{C}_{\perp}$ are lines of watershed. If one neglects the feedback term $V_{\mathrm{M}}$, the selection rule is simple :

For a given direction 1 of the wetting interface, the direction $\mathbf{n}_{l}$ of the molecules far from the contact line is situated in one of the valleys. As the molecules approach the contact line, their orientation evolves along gradient lines and «falls » into the minimum $\mathbf{n}_{\alpha}$ of that valley. The lines of watershed separate the different minima.

3.3 DETAILED FORM OF THE ANISOTROPIC INTERACTIONS $V_{\mathrm{D}}(\mathbf{n})$. - The most general form of the interaction potential $V_{\mathrm{D}}(\mathbf{n})$ is :

$$
V_{\mathrm{D}}(\mathbf{n})=\sum_{l, m} Q_{l m} Y_{l}^{m}(\mathbf{n})
$$

where $Y_{l}^{m}(\mathbf{n})$ are spherical harmonics. As for nematics $\mathbf{n} \equiv-\mathbf{n}$, only terms with even $l$ are different from zero.

3.3.1 $l=2$. - In the most general case $V_{\mathrm{D}}(\mathbf{n})$ for $l=2$, has only two minima $V_{\min }$, two maxima $V_{\max }$ and two saddle points $V_{\mathrm{s}}$. Let $\boldsymbol{\xi}, \boldsymbol{\eta}, \boldsymbol{\zeta}$ be three orthogonal directions defining the location of $V_{\min }$, $V_{\max }$ and $V_{\mathrm{s}}$. For example, in the figure $14 \mathrm{a}, \xi$ corresponds to the saddle point, $\boldsymbol{\eta}$ to the minimum and $\zeta$ to the maximum. There are five degrees of freedom allowed by five linearly independent coefficients $Q_{2}^{m}$. In the case of the symmetry $\mathrm{C}_{\mathrm{s}}$ one of the axes, say $\xi$, must be perpendicular to $\sigma$, so that only three degrees of freedom are left. In the vicinity of planar - asymmetric tilted - symmetric tilted transition, the anchoring direction evolves on a great circle $\mathrm{C}$, perpendicular to $\sigma$ and oblique with respect to $z$ (Fig. 12).

In the first approximation the direction of the $\zeta$ axis in the plane $\sigma$ is fixed as perpendicular to $\mathrm{C}$; only two degrees of freedom persist. 


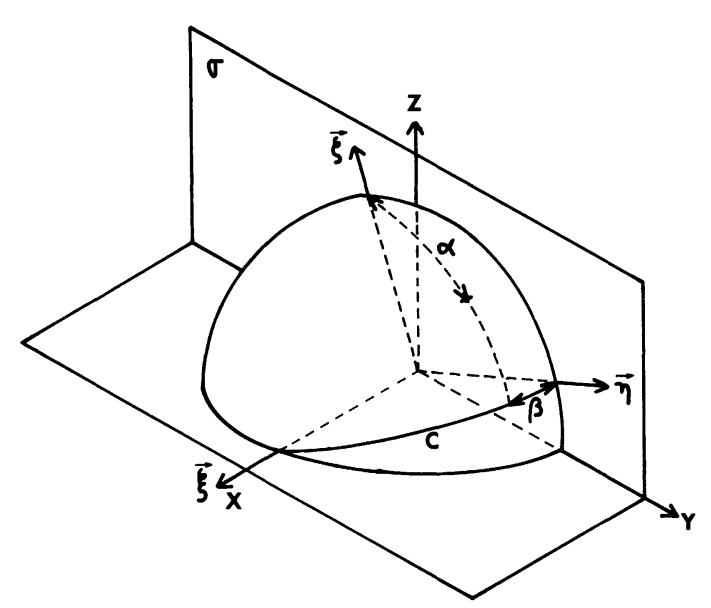

Fig. 12. - Definition of angles $\alpha$ and $\beta$.

Therefore, for the symmetry $\mathrm{C}_{\mathrm{s}}$ and in the vicinity of the planar-tilted transition, the potential $V_{\mathrm{D}}(\mathbf{n})$ can be represented as a linear combination of only two spherical harmonics :

$V_{\mathrm{D}}(\alpha, \beta)=Q_{20}\left(3 \cos ^{2} \alpha-1\right)+Q_{22} \sin ^{2} \alpha \cos 2 \beta$

where $\alpha$ and $\beta$ are defined in figure 12 .

Let $Q_{20}$ be positive. Then :

- for $Q_{22}<0$ the minima of $V_{\mathrm{D}}(\mathbf{n})$ are located on the $\boldsymbol{\eta}$ axis

- for $Q_{22}>0$ the minima of $V_{\mathrm{D}}(\mathbf{n})$ are located on the $\xi$ axis

- for $Q_{22}=0$ the potential $V_{\mathrm{D}}(\mathbf{n})$ is uniaxial and has a degenerate set of minima in the $(\boldsymbol{\xi}, \boldsymbol{\eta})$ plane.

Experimentally, the transition from the planar to the tilted anchorings occurs as a function of the evaporation angle $\varepsilon$. Thus one can suppose that :

$$
Q_{22}=\mathrm{C}\left(\varepsilon_{\mathrm{c}}-\varepsilon\right) \text { where } \varepsilon_{\mathrm{c}} \approx 66^{\circ} .
$$

In conclusion, if only $l=2$ terms are taken into account then the transition from the antisymmetric planar to the symmetric tilted anchoring is discontinuous. The coefficient $Q_{22}$ is the pertinent parameter of this transition.

3.3.2 $l=4$. - In order to obtain the intermediate asymmetric tilted anchoring, terms with $l=4$ must be taken into account. For $l=4$, nine spherical harmonics $Y_{4}^{m}(\mathbf{n})$ exist in the most general case. In the special case of $\mathrm{C}_{\mathrm{s}}$ symmetry this number is reduced to five. Finally, using the experimentally found evolution of $\mathbf{n}_{\alpha}$ on the great circle $\mathrm{C}$, only one fourth rank harmonic can be considered as pertinent :

$V_{\mathrm{D}}(\alpha, \beta)=Q_{20}\left(3 \cos ^{2} \alpha-1\right)+$

$+C\left(\varepsilon_{\mathrm{c}}-\varepsilon\right) \sin ^{2} \alpha \cos 2 \beta+Q_{44} \sin ^{4} \alpha \cos 4 \beta$

where $Q_{44}>0$.
For such a special choice of the potential $V_{\mathrm{D}}(\mathbf{n})$, its minima, if they are located in the $(\boldsymbol{\xi}, \boldsymbol{\eta})$ plane, must satisfy the condition :

$$
\begin{aligned}
\left(\frac{\partial V_{\mathrm{D}}}{\partial \beta}\right)_{\alpha=\frac{\pi}{2}}=-2 C\left(\varepsilon_{\mathrm{c}}-\varepsilon\right) \sin 2 \beta- & \\
& -4 Q_{44} \sin 4 \beta=0 .
\end{aligned}
$$

This equation has following solutions :

- for $\left|\varepsilon-\varepsilon_{\mathrm{c}}\right|<\frac{4 Q_{44}}{C}$, the minima are situated at

$$
\beta_{\min }= \pm \frac{1}{2} \operatorname{arc} \cos \frac{C\left(\varepsilon-\varepsilon_{\mathrm{c}}\right)}{4 Q_{44}}
$$

- for $\varepsilon-\varepsilon_{\mathrm{c}}>\frac{4 Q_{44}}{C}, \beta_{\min }=0$

- for $\varepsilon-\varepsilon_{\mathrm{c}}<-\frac{4 Q_{44}}{C}, \beta_{\min }= \pm \frac{\pi}{2}$.

They are plotted as a function of $\varepsilon$ in figure 13 .

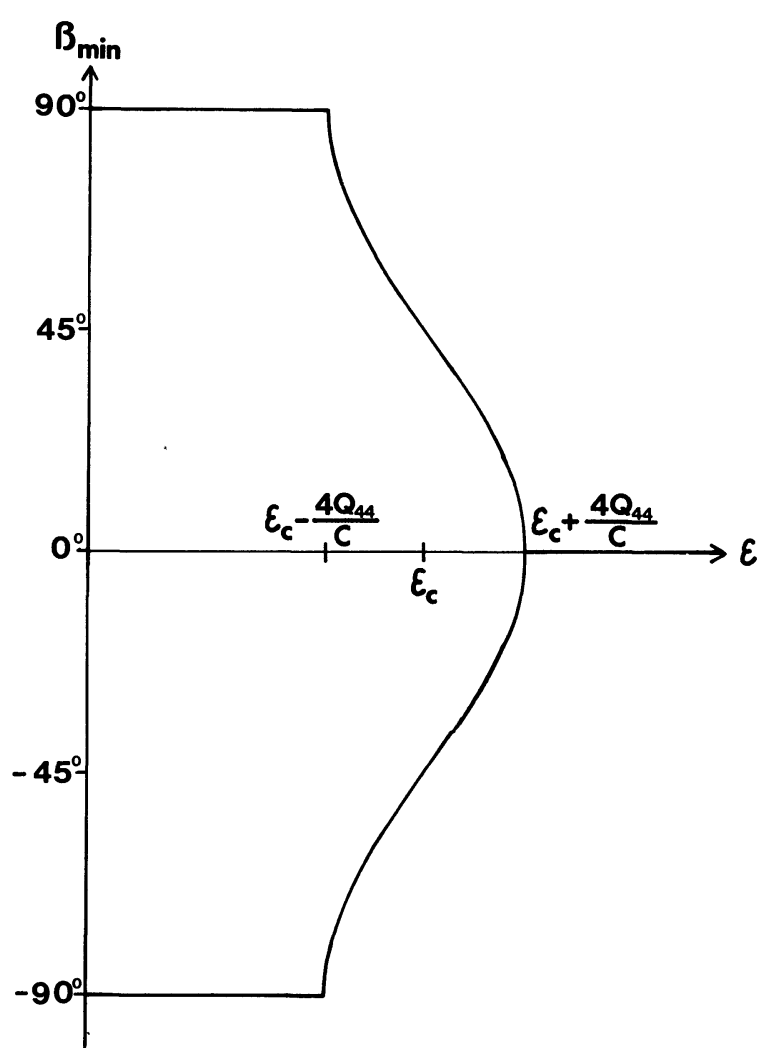

Fig. 13. - Azimuthal angle $\beta_{\min }$ versus incidence angle $\varepsilon$.

3.4 THEORETICAL SPHERICAL MAPS OF ANCHORING SELECTION. - Our criterion of anchoring selection, formulated in the section 3.2, is based on the lines of watershed, separating the different minima of the interaction potential $V(\mathbf{n})$. In the present case of the potential $V_{\mathrm{D}}(\mathbf{n})$, given by the equation (6), we can distinguish between three cases : 
a) symmetric tilted anchoring: for $\varepsilon-\varepsilon_{\mathrm{c}}>$ $4 Q_{44} / C$, there is only one separatrix. It is the great circle $\mathrm{C}_{\perp}$ perpendicular to $\sigma$ and located in the plane $(\xi, \zeta)$. It divides the sphere $(\delta, \psi)$ in two hemispheres corresponding to the two minima $\mathbf{n}_{1}$ and $-\mathbf{n}_{1} / / \boldsymbol{\eta}$

b) antisymmetric planar anchoring : for $\varepsilon-\varepsilon_{\mathrm{c}}<$ $4 Q_{44} / C$, there is also only one separatrix. It is the great circle $C_{\sigma}$, which divides the sphere $(\delta, \psi)$ in two hemispheres corresponding to the two orientations $\mathbf{n}_{1}$ and $-\mathbf{n}_{1} / / \boldsymbol{\xi}$;

c) asymmetric tilted anchoring: for $\left|\varepsilon-\varepsilon_{\mathrm{c}}\right|<$ $4 Q_{44} / C$ the two separatrixes $\mathrm{C}_{\perp}$ and $\mathrm{C}_{\sigma}$ are present. The sphere $(\delta, \psi)$ is divided in four quadrants, each corresponding to one of the four competing orientations $\mathbf{n}_{1},-\mathbf{n}_{1}, \mathbf{n}_{2}$ and $-\mathbf{n}_{2}$.

In figure 14 we show spherical plots of the potential $V_{\mathrm{D}}(\mathbf{n})$ illustrating the above three cases.

3.5 WeTtiNG TEXTURES DEDUCED FROM $V_{\mathrm{D}}(\mathbf{n})$. Such spherical maps of the anchoring selection can be used to predict the effects of a wetting of known geometry. For example, using the equation (3), the potential $V_{\mathrm{D}}(\mathbf{n})$ can be projected into the plane $(r, \psi)$ in the case of radially spreaded droplets. As shown in figure 15 , in this projection the separatrixes $\mathrm{C}_{\perp}$ and $\mathrm{C}_{\sigma}$ become, respectively a straight line and a closed curve similar to the cardioid.

As expected, these lines correspond to the walls, observed in the experimental wetting textures shown in figures 4,5 and 8.

There are however several details missing on the theoretical plots :

1) the cardioid wall, observed in our experiments has cusps at $\psi=0$ and $\Pi$ where it intersects the mirror plane $\sigma$;

2) in several droplets the cardioid wall makes large scale zig-zags (Fig. 5) ;

3) besides the cardioid wall, the observed wetting texture contains also crescent-like domains (Figs. 5 and 8) ;

4) other domains may be created inside the cardioid (Fig. 5b) ;

5) the diametral wall becomes erratic when it goes away from the center of spreaded droplets; at the periphery the anchoring selection is erratic.

Explanation of all these features requires several improvements of the model.

3.6 CORRECTIONS OF THE POTENTIAL $V(\mathbf{n})$. - As a wall in a wetting texture is a projection of a separatrix from the $V(\delta, \psi)$ plot onto the plane $(r, \psi)$, the missing details can be due either to an oversimplified form of the potential $V(\mathbf{n})$ or to incorrect projection rules.

There are at least three possible corrections of the potential $V(\mathbf{n})$ :

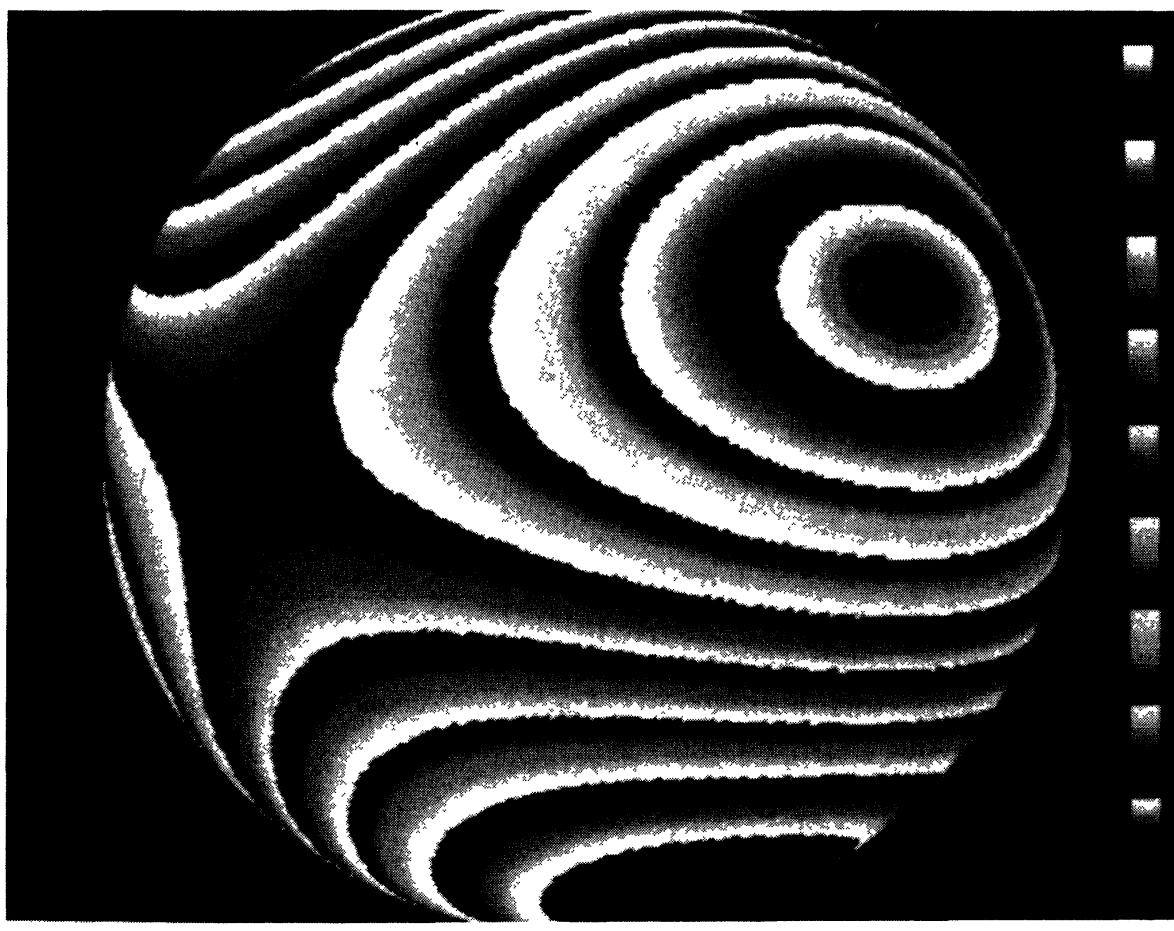

$+1$

a)

Fig. 14. - Spherical plots of the potential $V_{\mathrm{D}}(\mathbf{n})$ seen from the direction $\varphi=45^{\circ}, \theta=90^{\circ}:$ a) $\varepsilon-\varepsilon_{\mathrm{c}}>4 Q_{44} / C$; b) $\varepsilon-\varepsilon_{\mathrm{c}}<4 Q_{44} / C ;$ c) $\varepsilon=\varepsilon_{\mathrm{c}}$. 


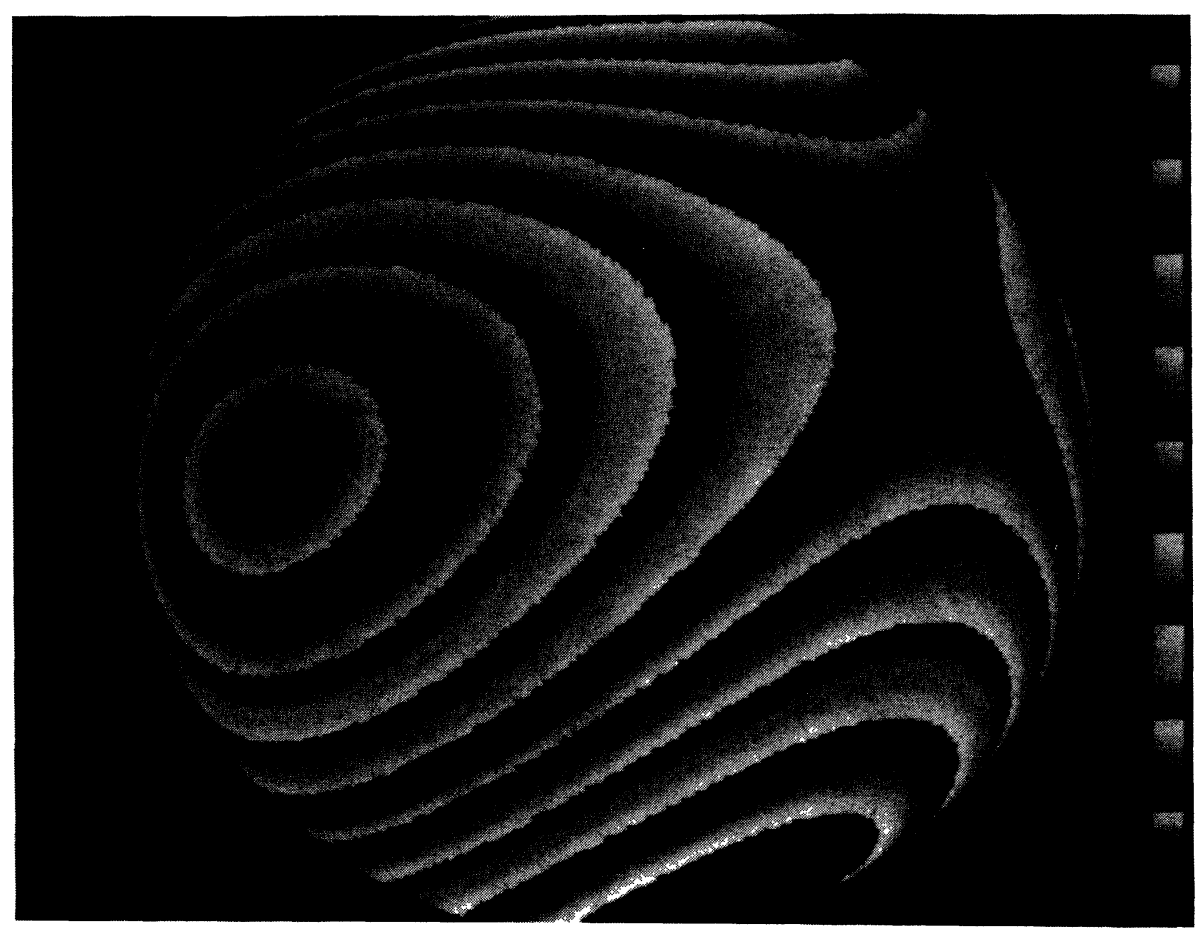

b)

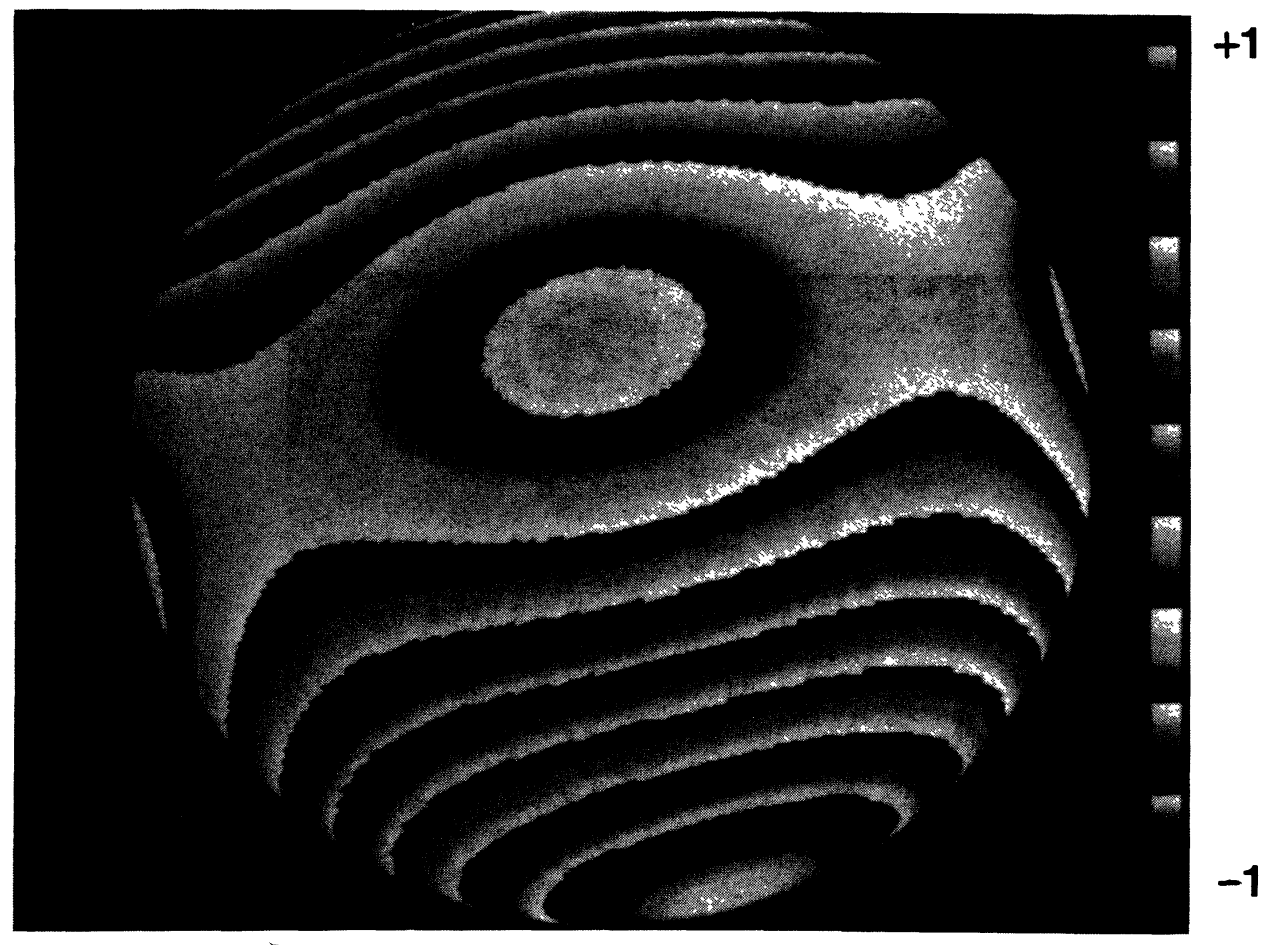

c)

Fig. 14 (continued)

1) other spherical harmonics allowed by the symmetry $\mathrm{G}_{\mathrm{s}}$ can be added to the potential $V_{\mathrm{D}}(\mathbf{n})$. They result in smooth modifications of wall shapes. The feedback term $V_{\mathrm{w}}\left(\mathbf{n}, \mathbf{n}_{\alpha}\right)$ can also be taken into account which would also modify the wall shapes;

2) due to the roughness of evaporated $\mathrm{SiO}$ films there must be a random part $V_{\mathrm{R}}(\mathbf{n})$ in $V(\mathbf{n})$. Due to this term, the separatrixes become erratic. Let $\Delta \psi \approx \Delta \delta$ be a measure of the fluctuations of the separatrix on the spherical plot $V(\delta, \psi)$. In the projection given by the equation (3) corresponding fluctuations $\Delta x, \Delta y$ in the plane $(r, \psi)$ diverge for $r \rightarrow \infty$. This explains the feature $5^{\circ}$ of the previous section. 


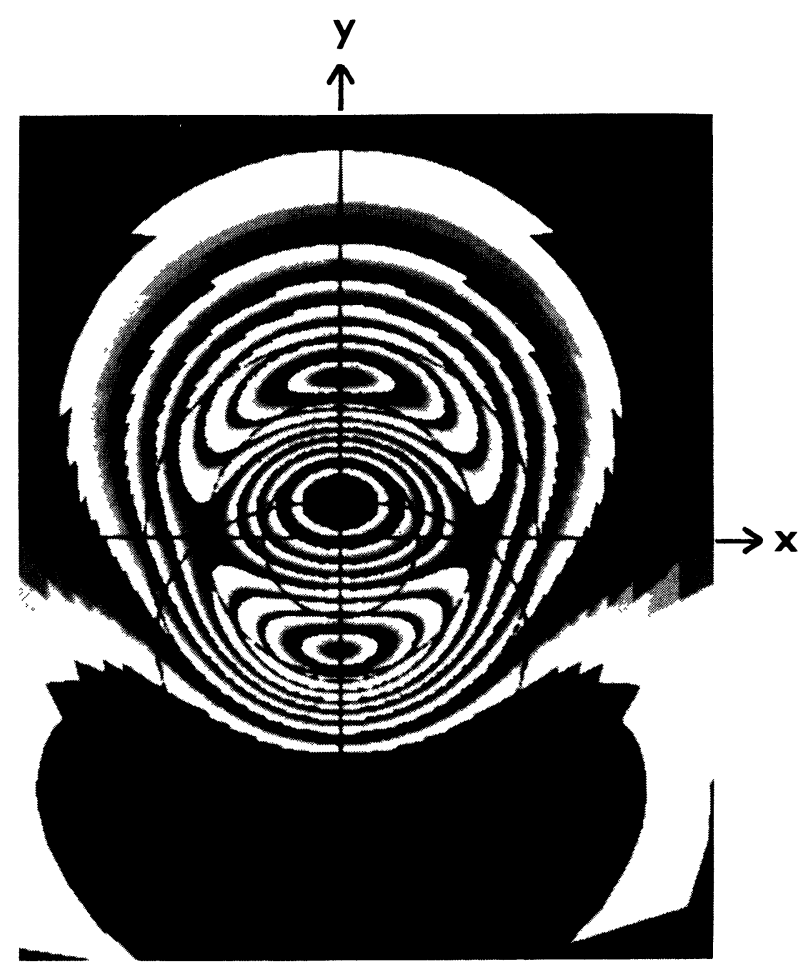

a)

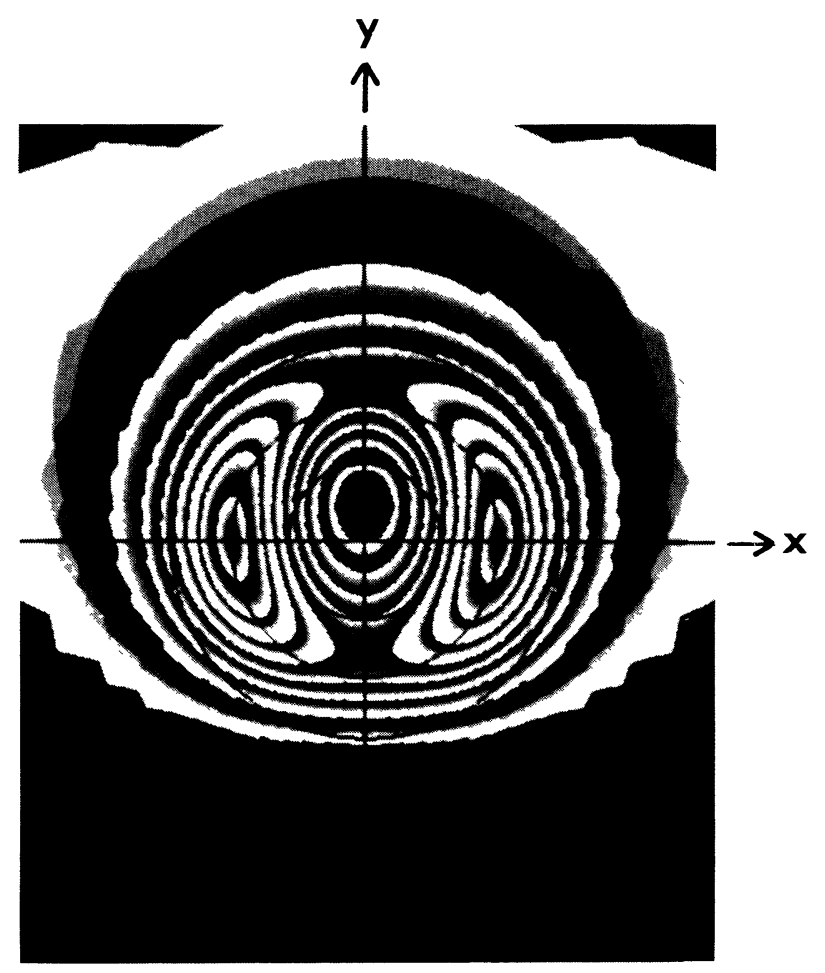

b)

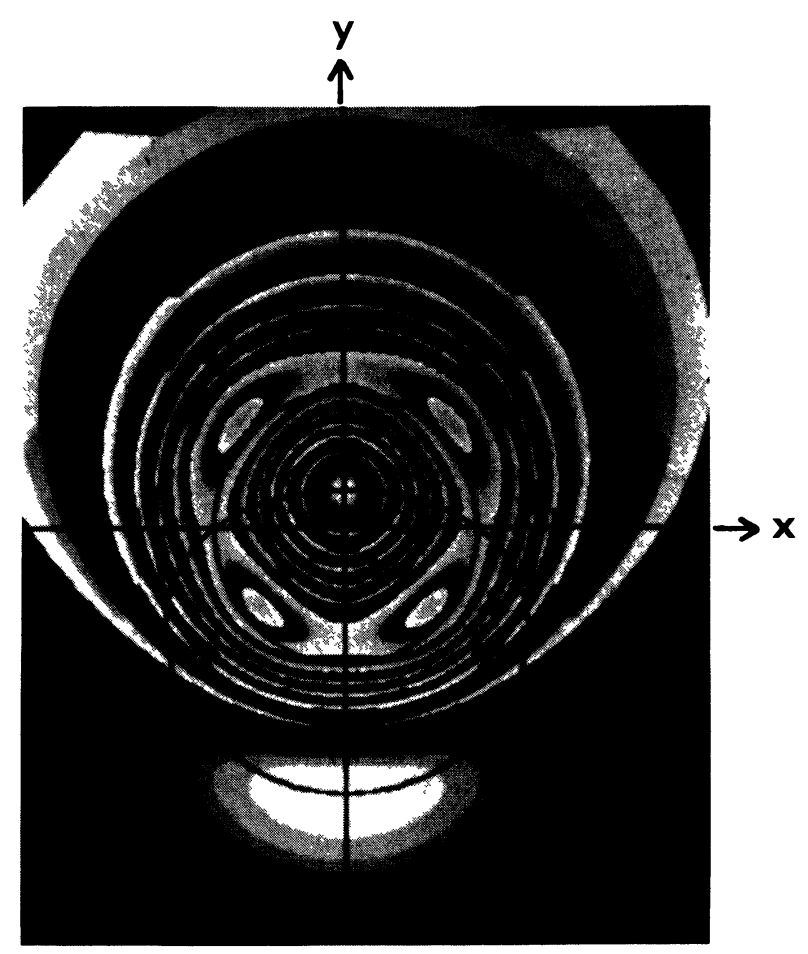

c)

Fig. 15. - Theoretically predicted droplet textures deduced from the projection of $V_{\mathrm{D}}(\mathrm{n}):$ a) $\varepsilon-\varepsilon_{\mathrm{c}}>4 Q_{44} / C$; b) $\varepsilon-\varepsilon_{\mathrm{c}}<4 Q_{44} / C$; c) $\varepsilon=\varepsilon_{\mathrm{c}}$. The center of the droplet is the intersection $\mathrm{O}$ of the $x$ and $y$ axes ; the three circles centered in $\mathrm{O}$ are the parallels $\theta=135^{\circ}, \theta=90^{\circ}, \theta=45^{\circ}$. 
3.7 CORRECTION OF PROJECTION RULES. - Our projection rule (3) was based on the assumption of a spherical form kept by the droplet during its spreading.

Deviations from the spherical shape of the droplet can arise for several reasons. The most obvious results from the method of depositing the drop on the substrate : the droplet hanging from the capillary is spherical but it becomes allongated vertically when it touches the substrate. When the link with the capillary breaks, strong capillary force driven oscillations of the droplet must arise. The effect of such oscillations is to perturb the projection rule (3); the contact angle $\delta(r)$ is no more a monotonically decreasing function of $r$ but shows oscillations as a function of $r$. Such oscillations result in zig-zag shapes of the cardioid wall and in the creation of the crescent like domains (features $2^{\circ}$ and $4^{\circ}$ in section 3.5). They can create other domains in the wetting textures (feature $3^{\circ}$ ).

\section{Conclusions and perspectives.}

Anisotropic solid substrates, which enable to create multistable anchoring conditions for liquid crystals, deserve special attention not only because of their possible applications in display technology but, first of all, because of a number of interesting physical questions, which they rise. All these questions can be classified in four chapters related to the four elements of the wetting process presented in the introduction.

The chapter devoted to the preparation of the dry substrates seems so far to be the most voluminous, nevertheless, there are only very few explicit attempts to create substrates for multistable anchorings. All such substrates can be classified following their symmetry $G_{s}$ and for each symmetry, all virtually possible types of anchorings can be classified using stars $\left\{\mathbf{n}_{\alpha}\right\}$ of equivalent anchoring direction. In the present paper we have considered, as an example, $\mathrm{SiO}$ films and shown that such films, when evaporated by a collimated $\mathrm{SiO}$ beam under oblique incidence, have $\mathrm{C}_{\mathrm{s}}$ symmetry and, for the range of evaporation angles $60^{\circ}<\varepsilon<72^{\circ}$, they create bistable asymmetric tilted anchoring in nematics. The process of evaporation under oblique incidence is related to a very interesting and actually very active theoretical topic - the ballistic aggregation [8]. Can the $\mathrm{C}_{\mathrm{s}}$ symmetry of the substrate be obtained by other means or, inversely, can the evaporated $\mathrm{SiO}$ films have other symmetries than $\mathrm{C}_{\mathrm{s}}$ ? In any case the simplest method to detect the type of anchoring seems to be the observation of the wetting textures in spreaded droplets which are true «finger-prints » of multistable anchorings. (Besides the $\mathrm{C}_{\mathrm{s}}$ symmetry, $\mathrm{C}_{2 \mathrm{v}}$ and $\mathrm{C}_{4 \mathrm{v}}$ seem to be good candidates for multistable anchorings).

The problem of the wetting textures, can be classified in the chapter devoted to the motion of the contact line but, as we have shown in the section 3 , the anchoring selection involves interactions with the dry and the already wet substrate. The nature of these interactions has to be elucidated. The anisotropic van der Waals interactions and elastic inteactions seem respectively to be good candidates.

Independently of their nature, the symmetry of these interactions is the predominant qualitative factor in the process of anchoring selection. In the present paper we have proved the reality of the anchoring selection during the wetting. Can a switching between coexisting anchorings be obtained « a posteriori », for example, by an action of external fields? Walls between the domains in the case of $\mathrm{SiO}$ substrates seem to be strongly pinned.

This last question could be rather adressed in an already rather voluminous chapter devoted to the properties of wet substrates.

\section{Acknowledgments.}

We thank Mr. Collard of the Institut d'Optique, Orsay, for making the E-shaped glass slides and P. Oswald for letting us use his electromagnet.

\section{References}

[1] For a review on wetting see : DE GENNES, P. G., Rev. Mod. Phys. 57 (1985) 827.

[2] For a review on anchoring see : Cognard, J., Mol. Cryst. Liq. Cryst. Suppl. 1 (1982).

[3] Uchida, T., Ohgawara, M., Wada, M., Jpn J. Appl. Phys. 19 (1980) 2127.

[4] Monkade, M., Boix, M., Durand, G., a) rapport de stage DEA de Physique des Liquides (1984); b) Order electricity and oblique nematic orientation on rough solid surfaces, submitted to Europhys. Lett.

[5] Jéróme, B., Pieranski, P., Boix, M., Bistable anchoring of nematics on $\mathrm{SiO}$ films, submitted to Europhys. Lett.
[6] E8 is produced by $\mathrm{BDH}$.

[7] Kooy, C., Nieuwenhuizen, J. M., Basic Problems in Thin Film Physics, Eds. R. Niedermayer and H. Mayer (Vandenhoeck and Ruprecht, Göttingen) 1966 p. 181.

[8] Meakin, P., Jullien, R., J. Phys. France 48 (1987) 1657.

[9] Janning, J., Appl. Phys. Lett. 21 (1972) 173.

[10] Guyon, E., Pieranski, P., Boix, M., Lett. Appl. Eng. Sci. 1 (1973) 19 ;

Urbach, W., Boix, M., Guyon, E., Appl. Phys. Lett. 25 (1974) 479.

[11] Huh, C., Scriven, L., J. Colloid Interface Sci. 35 (1971) 85. 